\title{
Kratom- A Lethal Drug On The Rise
}

\begin{abstract}
In Malaysia, Dutch botanist Pieter Korthals in 1831 first discovered Kratom or Mitragyna speciosia. Kratom is derived from the Nitragyna speciose korth, a tropical forest tree found in Malaysia, Thailand and Myanmar. The trees' leaves contain contain psychoactive opioid compounds, consumed for thousands of years. Kratom contains alkaloids that bind to opioid receptors, with an opioid drug structure. Kratom induces euphoria, and in lower doses acts similar to a stimulant by increasing energy, alertness, while in higher doses it induces sedation. Kratom is purportedly used for anxiety, depression, inflammation, libido. Importantly, given opioid activity, it suppresses opioid withdrawal. With regular use, Kratom is associated with dependence and addiction.

Kratom is gaining increasingly popularity especially among the young in the US population and is causing rising numbers of ER visits, calls to poison control centers and even deaths related to multiple causes.

There are currently very limited clinical studies available that demonstrate safety and efficacy in humans. Kratom The FDA has not approved Kratom for medical use, and publishes warnings regarding its toxicity. Available information is mostly based on reports from users or animal models. Case reports involving the use of Kratom are concerning and alarming.
\end{abstract}

Kratom is classified as an herbal supplement and therefore easily available to the user as it is sold in convenience stores, online or gas stations. At this point, it is a dangerous drug with opioid activity, freely available without controls for safety.

This article is a systematic review of literature on the current available data on Kratom guided for physician and clinician education and to raise awareness about Kratom as a substance.

Utilized sources were Pubmed, Ovid, Medline, Psychlnfo, and EMBASE.

\section{Introduction}

Kratom is a natural substance stemming from the Mitragyne speciosia tree in Southeast Asia and has been used for thousands of years [1,2]. In Asian countries it was used as a remedy for diarrhea, pain, hypertension and cough. Also, it has been used in religious ceremonies [1-3], (Figure 1).

Kratom can be ingested in various routes, traditionally chewed but also smoked or strained in teas. The leaves have a very bitter taste that can be masked by adding sugar or honey in juices or teas. As an alternative, lemon juice can be added to facilitate the extraction of the plant alkaloids. In Malaysia, the pill form is known as mandarin and smoked in long bamboo pipes $[1,4]$.

In Malaysia and Thailand Kratom was originally used to treat intestinal infections, muscle pain, diarrhea or coughing. Other indications were energy booster for workers to work in the fields in
Journal of

Addiction \& Prevention

\section{Oberbarnscheidt $\mathrm{T}^{1^{*}}$, Miller NS ${ }^{2}$}

${ }^{\prime}$ Department of Psychiatry, University of Pittsburgh, USA ${ }_{2}^{2}$ Professor of Psychiatry, Augusta University, current president of Health Advocates PLLC, East Lansing, MI, USA

\section{*Address for Correspondence}

T. Oberbarnscheidt, MD PhD, Western Psychiatric Institute and Clinic, Department of Psychiatry, University of Pittsburgh, Pittsburgh, PA, Email: Oberbarnscheidtt@upmc.edu

Submission: March 15, 2019

Accepted: March 26, 2019

Published: March 28, 2019

Copyright: () 2019 Oberbarnscheidt T. This is an open access article distributed under the Creative Commons Attribution License, which permits unrestricted use, distribution, and reproduction in any medium, provided the original work is properly cited.

hot and humid climate $[1,2]$.

The use of Kratom has now spread all over the world. In the US it is mostly used for its stimulant effect, to enhance performance and also to self-treat opioid withdrawal symptoms as a cheaper alternative to Buprenorphine [5-8].

In recent years Kratom has become increasingly popular in the US. About 40 million Americans are current using Kratom recreationally [9]. Most nationally conducted surveys on drug and alcohol use don't ask about Kratom use, so the actual number of users might be much higher, in particular in adolescents since it is easily accessible and legal which might falsely be perceived as "safe" and "just natural" [10].

Calls to US poison centers related to Kratom exposure increased from 26 calls in 2010 to 263 calls in 2015 [11]. 36 deaths were reported to the FDA in 2017. Kratom appears to be a drug increasingly used in the US and published case reports and toxic effects are emerging public health threat. Many health care providers have never heard about Kratom as a substance, which bears a need for education and

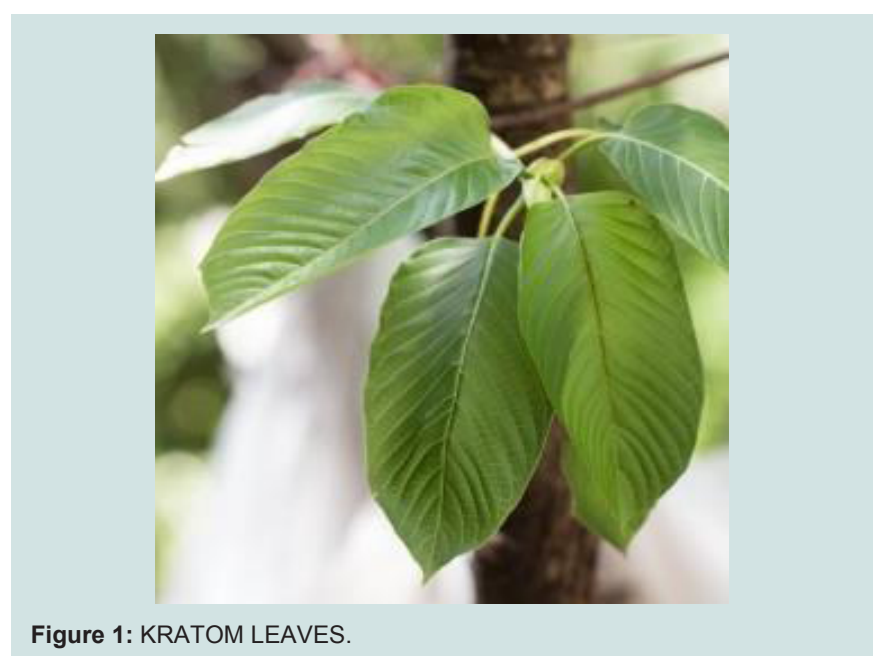


awareness [12].

Kratom's pharmacological properties are similar to those of opioids but unlike opioids it is not a federally regulated substance [12]. There are increasing number of case reports with Kratom intoxications and withdrawals that are challenging for the physicians. Kratom is not a very commonly known substance and is also difficult to detect as it does not cause positive findings in urine drug screens [13]. The assessment relies on the history from the patient or specific immune-assay tests [14]. The treatment can consist of symptomatic treatment with Clonidine but also with Suboxone. There are several cases reported of Kratom withdrawal and dependence [15].

According to the Drug Enforcement Agency Kratom is a legal herbal product and available without restrictions in many states [12]. It is not a federally controlled substance and listed with the FDA as "drug of concern". Some states banned the sale and possession of Kratom, those are Indiana, Tennessee, Wyoming, Florida, Alabama, and Wisconsin [16].

Is Kratom a Substance that is Safe to be ordered off the Internet or be purchased at the local convenience store?

\section{Legal status}

Thailand scheduled preparations containing M. speciosa as early as 1943. In 1979 the Thai government moved Kratom to schedule 5 of the Thai Narcotics Act which makes it illegal to buy, sell, import or possess Kratom [17]. Malaysia tolerated Kratom until 2003 when it placed the substance under the Poison Act, making the sale of Kratom illegal [18]. Countries that still allow the sale and cultivation are Indonesia which does sell Kratom on a large scale to North America as well as European countries [19]. Denmark, Poland and Sweden control the import of Kratom, but Germany and the US don't control the import of this substance on a federal level [20].

In the US, the Drug Enforcement Administration has placed Kratom on the list of drugs with "chemicals of concern". From a legal standpoint, Kratom is regulated as an herbal product under US law and US Food and Drug Administration and US Drug Enforcement Administration (DEA) policies [12]. In 2016 the DEA attempted to move Kratom to a schedule I substance, but public protest throughout the US resulted in rejection of that plan. A letter was sent to Barack Obama in September 2016 containing 130,000 signatures to support the availability of Kratom for recreational use [21]. Within 2 month the DEA withdrew the proposal to move Kratom to a scheduled substance.

Six States have banned the distribution and sale of Kratom but in the majority of states within the US, Kratom is available through Internet or local convenience stores, smoke shops and even gas stations [16]. Online, Kratom can be purchased as a plant itself or in form of dried leaves or preparations in drinks $[1,3]$.

\section{Pharmacology of Kratom}

Origin and mechanism of action: Kratom (Mitragynia speciosa korth) is a member of the Rubiaceae family, the same family that includes coffee [1-3]. It is also known under the name Thang, Kakuam, Thom, Ketum and Biak [22]. The fresh or dried leaves can be chewed, smoked or consumed orally. It can be brewed with hot water and made into syrup or powered and pressed into pill forms which are the most convenient form to distribute online [23].

The pharmacological effects of Kratom are dose dependent, small doses $1-5 \mathrm{~g}$ of raw leaves can cause stimulant like effects, while higher doses 5-15g can produce sedation and opioid like effect [24].

Animal studies and reports from users have shown that Kratom does act as antipyretic, antidiarrheal, euphoric anti-depressant as well as anxiolytics. It has shown to have properties as immunosuppressant, appetite suppressant and anti-diabetic medication [25].

Kratom is a drug that acts in the central nervous system. It is very lipophilic and easily crosses the blood brain barrier which causes the onset of effects to occur quickly and potent [26].

Kratom contains 2 main alkaloids: mitragynine and 7-hydorxymitragynine (1-3). Both act as opioid agonist. It does bind on mu-, delta as well as kappa opioid receptors [27]. However, the classification of kratom as and "opioid" is based on its mechanism of action and not on its molecular structure but more on it's pharmacological effects.

Mitragynine is 13 times more powerful than morphine however the concentration is very low about $0.2 \%$ in Kratom. Twenty leaves contain about $17 \mathrm{mg}$ of mitragynine [28].

Mitragynine is also known as an adjunct to synthetic cannabinoids such as K2 products [29]. There are also other pharmacological active ingredients found 7-hydroxymitraggynine, paynantheine, speciogynine and 20 other substance that are not well understood yet [28].

The effects of Kratom on the central nervous system as well as systemic effects can be inhibited or blocked by opioid antagonists [27].

Kratom also acts as an antagonist on Calcium-channels, inhibiting the neurotransmitter release from the vas deferens [30].

In addition is has agonist activity on alpha-2 receptors that avert opioid withdrawal symptoms and may work similar to other symptomatic treatments for opioid withdrawal for example Clonidine [31].

The stimulant like effect of kratom is mostly contributed to the antagonism on 5-HT2A receptors and it's effect is described very similar to the effect of cocaine. Studies (Kumarnsit) have shown that the dorsal raphe nucleus is a major binding site of Kratom in the central nervous system [32].

The onset after chewing the leaves is about 5-10 min and lasts approximately 2-5 hours.

The average clearance of mitragynine has been measures as 1.6 $\mathrm{L} / \mathrm{h}$, the elimination half-life is reported as 3.85 hours, and the total clearance is $6.35 \mathrm{~L} / \mathrm{h} / \mathrm{kg}$. In laboratory tests in rats, mitragynine could still be detected in the rats 24 hours after oral administration of Kratom [33].

The detection of Kratom requires specialized tests:

Ultra-high-performance liquid chromatography and highperformance liquid chromatography- tandem mass spectrometry methods have been successfully used in monitoring the major 
alkaloids and metabolites found in urine following Kratom use [34].

\section{Kratom toxicity}

Kratom is associated with several severe toxic effects including hypertension, nephrotoxicity, psychosis, seizures, and hepatotoxicity. The risks of long-term use of Kratom are currently unknown. There are no pharmacological or pharmacokinetic studies on humans published or studies on drug interactions involving Kratom [35].

Kratom has shown in animal studies to affect the endocrine system and suppress TSH similar to the effect of morphine on the thyroid or stress related effects on the patient [36]. The liver toxicity can range from mildly elevated liver enzymes to acute liver failure. A case report from Kapp reported acute liver failure with jaundice and pruritis following 2 weeks of ingestion of Kratom [37].

Also, neurotoxic effects of Kratom have been reported. During the acute intoxication, there are cases of seizures reported from individual case reports which also have been confirmed in animal studies. Roche et al published a case report about a woman who presented with a status epilepticus requiring intubation and ICU care after ingestion of Kratom off the internet 24 hours prior [38]. The exact mechanism is currently unknown. There are theories that the mitragynines and their metabolites can lower the seizure threshold similar to atypical opioids such as Tramadol or Meperidine. Kratom has been shown to be neurotoxic in in vitro studies on neuronal cells. The neurotoxicity can be reversed or prevented by administration of Naloxone [38].

Respiratory depression is not reported consistently in studies and appears to be dose dependent but death from overdoses have been reported in the US as well in other countries [39]. The lethal dose of Kratom in rats has been identified as $200 \mathrm{mg} / \mathrm{kg}$. There is currently no exact lethal dose known for humans. Overdoses have been reported in the setting of long-term use and exposure as well as acutely after first time ingestion [40].

Kratom when used during pregnancy has shown to be associated with complications in pregnancy and has caused cases of neonatal abstinence syndrome in the newborn [41].

\section{Is Kratom an addictive substance?}

Kratom's pharmacological properties and reported rewarding effects bear a significant risk of the development of an addiction. Both the stimulant effect as well as the opioid like effect can lead to compulsive use, use despite the negative consequences and regardless or the decline in psychosocial functioning.

Animal studies in mice have shown that the rewarding properties require an overtime dose increase to continue to have the same effect [42]. Which would implicate the development of tolerance in these mice after repetitive exposure of the substance? Individual reports from Kratom users confirmed this presence of tolerance but there is no controlled study in humans available to demonstrate this.

In early stages, the Kratom users are mostly able to fulfill their work requirements and psychosocial functioning but the prolonged use bears the risk of psychiatric problems and physical impairments that make this difficult or impossible, which is very comparable to other substances listed in the DSM V as substance use disorders.

Even though Kratom is not an acknowledge substance to cause addiction according to DSM V it does fulfill the equivalent criteria listed for opioid use disorder in some patients [43].

There are few case reports published that demonstrate the development of addiction is possible, for example a 37-year old teacher who tried Kratom for fibromyalgia and then consumed it compulsively in increasing amounts, and despite the negative effect on her marriage she was unable to cut back on the use without any medical assistance and professional withdrawal treatment [44]. The physiological and psychological dependence as well as tolerance and withdrawal are also reported in case reports as well $[45,46]$ Table 1.

Numerous reports have shown that the discontinuation of Kratom causes moderate withdrawal that consists of symptoms nausea, vomiting, diarrhea, lacrimation of eyes, rhinorrhea, restlessness, jerky limb movements, agitation, insomnia, decreased appetite, aggression as well as depression, anxiety similar to opioid withdrawal symptoms. The length of withdrawal can be prolonged and potentially last for more than a week [47].

In animal studies, the withdrawal symptoms could be induced by administration of Naloxone and was shown to be reproducible [48].

The use of Kratom is more difficult to detect and monitor since Kratom cannot be detected in regular drug screens. In order to measure

Table 1: DSM Criteria for opioid use disorder

\begin{tabular}{|c|}
\hline Table DSM - 5 Diagnostic Criteria for Opioid Use Disorder \\
\hline Opiods are taken larger amounts or duration than intended \\
\hline Persistent desire/ unsuccessful efforts to cut down or control opioid use \\
\hline A great deal of time is spent obtaining, using or recovering from the effects of opio \\
\hline Craving \\
\hline Recurrent use of opioid results in failure to fullfill major role obligations at work, school, \\
\hline $\begin{array}{l}\text { Continues use despite knowledge of having a persistent or recurrent opioid-related physical or psychological problem tha } \\
\text { use }\end{array}$ \\
\hline Tolerance $^{a}$ \\
\hline withdrawn ${ }^{b}$ \\
\hline Severity: Mild: 2-3symptoms, Moderate- $4-5$ symptoms, severe:>= 6 symptoms \\
\hline
\end{tabular}

aThe Information above is only an overview of the criteria used. Consult the DSM_5 before making a diagnosis.

${ }^{\mathrm{b}}$ Note : This criterion is not considered to be met for patients taking opiods solemnly under appropriate medical supervision 
mitragynine serum concentrations in rats and humans, technologies high performance liquid chromatography with ultraviolet detection is needed [49].

The current accessibility of Kratom makes the supply for development of addiction easy as Kratom is cheap and easy to obtain: Kratom is easily purchased through the internet and much cheaper than heroin and methamphetamines. Kratom Extract 50x (5 grams) cost $\$ 34.99$, comparison the Ultra enhanced form is more expensive, priced 5 grams $\$ 45.99$. The price for an entire Kratom plant is around $\$ 50$ according to my internet searches.

\section{Reported indications for Kratom use}

Kratom effects on humans are not well studied and understood yet. Early reports were made by Wray in 1907 [50] [51]. Most studies that are available about Kratom are animal studies from rodents (mice and rats).

Pain: Animal studies have shown that Kratom prolongs the latency to nociceptive or painful stimuli. In 2010 Sabetghadam et al compared the anti-nociceptive effect of Kratom with Morphine in rats and noticed a significant increase in pain response latency by administering the "tail-flick- test" to the rats. Also, the group noticed that the analgesic effect of Kratom can be potentiated by co-administration of caffeine. This anti-nociceptive effect was found with oral administration of Kratom as well as intra-cerebroventricularly. In addition, it was noted that the effects of Kratom on the pain perception could be blocked with co-administration of Naloxone [52].

Other animal studies showed and anti-novice-time effect, which is important in pain signaling and pain response of even 13 times more potent than morphine [53].

An additional benefit from Kratom over morphine is that is also acts as a muscle relaxant which has been shown in rats. It blocks the nerve conduction as well as amplitude and action potential [53].

Inflammation: Kratom carries some anti-inflammatory properties: Kratoms major active compound, Mitragynine, inhibits cyclooxygenase (COX) 1 and 2 isoenzymes and in COX 2 mRNA and protein expression as well as the release and production of Prostaglandin PGE2 which is one of the strongest inflammatory mediators [25].

In rodents, It has been shown that Kratom inhibits the early phase of paw edema development in the first 3 hours after challenge. In addition, it is found to inhibit the development of granuloma tissue through particular macrophages as well as fibroblasts in the acutely inflamed tissue. Kratom stimulates tissue repair, increases vascular permeability in supports healing and immunity [25].

Kratom is found to have anti-microbacterial properties against bacteria and has been tested against Salmonella typhi and Bacillus subtilis which would explain why Kratom has been used traditionally to treat gastro-intestinal infections [54].

Self-treatment of opioid withdrawal: The first cases of opioid substitution with Kratom were described by Low in the 1800's [2]. Kratom is frequently used for opioid withdrawal symptoms. Blots in the internet discuss and advise how to obtain and use it. There is no medical guided treatment for opioid withdrawal or treatment prescribed by a physician [31].

Kratom's compound Mitragynine is acting as an agonist on postsynaptic alpha 2 receptors similar to Clonidine which is regularly used for opioid withdrawal symptoms. Unlike Clonidine it does improve withdrawal symptoms but at the same time potentiates the opioid effect of the initially ingested opioid and also potentiates effects from other sedative or hypnotic substances which does increase the risk of sedation [31].

There are currently no studies available investigating the effect of Kratom on opioid withdrawal symptoms in either human or animals.

Weight loss medication: Animal studies in rats have shown that acute as well as chronic administration lead to reduced food and water intake and weight loss. Kratom influences the transit time of water and food in the way of slowing the process down [55]. This effect is mostly present in lower doses as Kratom does act similar as a stimulant [24].

Other animal models show that Kratom does increase the glucose uptake into the cell and increases the protein transporters for glucose [55].

Unlike other substances that induce weight loss, it does not affect the secretion or levels of cholecystokinin [56].

There are no human studies available that show the success rate of weight loss or the associated long-term effects and adverse reaction. There are many online forums of users that discuss the use of Kratom for the indication of weight loss as a so called "health supplement".

Kratom use for cognitive enhancement: The use of Kratom influences short-term learning in a positive way, so enhances shortterm learning but does not improve any long-term consolidation of learned material [57-59]. Further, animal tests have shown a delay in passive avoidance learning. These effects of Kratom are facilitated by Kratom's inhibition of neuronal potentials in the hippocampus. Compared to heroin users, chronic Kratom users have shown to perform poorly on visual-spatial recognition tests [60].

\section{Discussion}

Kratom has been used in Asian countries as a natural remedy for thousands of years. However, in the US, Kratom is becoming more and more popular as a recreational drug for its stimulant as well as opioid-like effect. The pharmacology and active ingredients are not fully understood and available human research studies on Kratom are very limited.

The increasing number of calls to the poison control centers show, Kratom is not harmless even though it is a natural substance. Opium is derived from a natural plant and poppy seeds as well and is clearly a federally schedule 1 controlled substance. The term natural should not be seen equal to harmless.

Should Kratom be available without any restrictions or should Kratom become a schedule 1 substance controlled by the DEA or should there be a Medical Kratom similar to the Medical Marijuana. The current trend and failed attempt to federally control Kratom bears risks for the US population. 
Further studies are needed to completely understand Kratom and its effects.

Research with Kratom as an herb is complicated due to the inconsistencies in the substance which Kratom product should be used and become evaluated? How should the standardization occur in a substance with mixed compounds?

Another factor is that Kratom is not well known to many physicians. Increased education is needed to provide improved assessment of situation as well as improve treatment. Since Kratom does not provide positive results in urine drug tests, the diagnosis relies and the subjective history and specific questioning by the physician.

Random screening tests for substance use disorders should include questions regarding Kratom use. Also, it should be subject to discussion, whether to include Kratom as a use disorder in the DSM V.

\section{Conclusion}

Kratom is a substance that has been available for a long time especially in the Asian areas that has certainly potent medical properties. However more research and clinical studies are needed to further investigate the properties of Kratom and toxicities. The reported toxic effects of Kratom that have been confirmed in animal studies are concerning.

The pharmacology of Kratom appears very fascinating how it can act as a stimulant in low doses but then more as an opioid with sedation in higher doses. Not all chemically active ingredients are yet identified. There are many details to be learned about Kratom. Kratom might have some medical properties but unlike FDA approved medications, one sample is not the compatible with the next as the concentrations of active ingredients are varying from plant to plant.

There are parallels to the current trend and use of Kratom and Marijuana or so called "Medical Marijuana" these days. Those natural substances are in the general public seen as harmless and without major concerns. The legal availability makes Kratom and marijuana socially acceptable and is also easily accessible for the adolescents. There is no data available yet to look at the role of Kratom as a gateway drug and link to other consecutive substances. This is partially the case because only a very small fraction of kratom users reach out for medical treatment and gets even identified. This has been a problem among substance use disorders in general for decades. Public education is a helpful tool to reach out and eventually prevent further damage. The increasing number of case reports and contact with patients in emergency rooms or poison control centers are alarming to raise a concern to further regulate the availability of Kratom and consider a change to federally controlled substance.

The current trend and associated risk as well as the economical burden for medical care of Kratom users, missed worked days and overall reduced psychosocial functioning are alarming.

With the current legal situation and tolerance to these natural substances, Kratom and also Marijuana we are facing a trend in society and a high degree of tolerance to allow and legalize impairment. Alcohol as a licit substance shows similar impairment however is easily detectable in breathalyzer tests and will cause consequences when used at workplace or hazardous situations for example traffic. Kratom on the other hand does not show in standard drug screens or tests. The current legal state allows everybody including bus drivers, physicians and law enforcement officers to use it with all the negative consequences.

More public awareness and physician education is needed regarding Kratom. Until further and more detailed data on the pharmacology and toxicity of Kratom in particular in humans is available, stricter control and regulation would be safer for the US population.

\section{References}

1. Dizon DS (2010) What's the Harm. Oncologist. 22:1006-1007.

2. AH Beckett, EJ Shellard, JD Philipson, CM Lee (1965) Alkaloids from Mitragyna speciosa (Korth), J of Pharmacy and Pharmacol.17:753-755.

3. Cinosi E, Martinotti G, Simonato P, Darshan Singh, Zsolt D et al. (2015) Following "the Roots" of Kratom (Mitragyna speciosa): The evolution of an enhancer from a traditional use to increase work and productivity in Southeast Asia to a recreational psychoactive drug in Western countries. Bio Med Res Int: 968-786.

4. HF Macmillan (1991) Tropical Planting and Gardening, (6th ed.), Malayan Nature Society, Kuala Lumpur.

5. Scholz and Eigner (1983) To the knowledge of natural hallucinogens, Pharmaceuticals in Our Time, 12: 75-79.

6. Utar Z, Majid MI, Adenan MI, Jamil MF, Lan TM (2011) Mitragynine inhibits the COX-2 mRNA expression and prostaglandin $E(2)$ production induced by lipopolysaccharide in RAW264.7 macrophage cells, J of Ethno pharmacol 136: 75-82.

7. Ahmad K, Aziz Z (2012) Mitragyna speciosa use in the northern states of Malaysia: a cross- sectional study. J of Ethno pharmacol 141: 446-450.

8. Suwanlert S (1975) A study of kratom eaters in Thailand. Bulletin on Narcotics, 27: 21-27.

9. Drug Enforcement Administration, (2013) Office of Diversion Control, Drug \& Chemical Evaluation Section, Springfield, VA: US Government Printing Office. Kratom (Mitragyna speciosa korth).

10. Ward J, Rosenbaum C, Hernon C, McCurdy C, Boyer C (2011) Herbal medicines for the management of opioid addiction: safe and effective alternatives to conventional pharmacotherapy? CNS Drugs 25: 999-1007.

11. National Institute on Drug Abuse (2016) Drug Facts: kratom. Bethesda, National Institute on Drug Abuse.

12. National Center For Complementary and alternative Medicine (NCCAM). Guidance for industry on complementary and alternative medicine products and their regulation by the Food and Drug Administration (2006)

13. Le D, Goggin MM, Janis GC (2012) Analysis of mitragynine and metabolites in human urine for detecting the use of the psychoactive plant kratom? J Anal Toxicol 36: 616-625.

14. Sukrong S1, Zhu S, Ruangrungsi N, Phadungcharoen T, Palanuvej C, et al. (2007) Molecular analysis of the genus Mitragyna existing in Thailand based on rDNA ITS sequences and its application to identify a narcotic species: Mitragyna speciose. Biol Pharm Bull 30: 1284-1288.

15. Wing N. Does Kratom really kill? Officials aren't telling use the whole story. Authorities claim the popular herbal drug poses potentially deadly risks, but there are big problems with their evidence. Huffington Post, Healthy Living,

16. Vicknasingam B, Narayanan S, Beng G, Manor SM (2010) The informal use of ketum (Mitragyna speciose) for opioid withdrawal in the northern states of peninsular Malaysia and implications for drug substitution therapy. Int $\mathrm{J}$ of Drug Policy 21: 283-288.

17. Tanguay $P$ (2011) Kratom in Thailand. Legislative reform of drug policies 13: $1-16$. 
18. European Monitoring Centre for Drugs and Drug Addiction. Kratom (Mitragyna speciosa) drug profile.

19. J. Hillebrand, D. Olszewski, R. Sedefov. Legal highs on the Internet. Hogan Lovells. Letter to acting DEA Administrator. Chuck Rosenberg.

20. Jansen KL, Prast CJ (1988) Ethnopharmacology of kratom and the Mitragyna alkaloids. J of Ethnopharmacol 23: 115-119.

21. Scholz D, Eigner D, (1983) To the knowledge of natural hallucinogens. Pharmaceuticals in our time 12: 75-89.

22. Jansen KL, Prast CJ (1988) Psychoactive properties of mitragynine (Kratom). $\mathrm{J}$ of Psychoactive Drugs 20: 455-457.

23. Shaik Mossadeq WM, Sulaiman MR, Tengku Mohamad TA, Chiong HS, Zakaria ZA et al. (2009) Anti- inflammatory and antinociceptive effects of Mitragyna speciose Korth emthanolic extract. Med Princ Pract 18: 378-384.

24. Adkins JE, Boyer EW, McCurdy CR (2011) Mitragyna speciosa, a psychoactive tree from Southeast Asia with opioid activity. Curr Top Med Chem 11: 1165-1175.

25. Macko E, Weisbach JA, (1972) Douglas B. Some observations on the pharmacology of mitragynine. Arch Int Pharmacodyn Ther 198: 145-161.

26. Yamamoto LT, Horie S, Takayama H, Aimi N, Sakai S, (1999) Opioid receptor agonistic characteristics of mitragynine pseudoindoxyl in comparison with mitragynine derived from Thai medicinal plant Mitragyna speciose. Gen Pharmacol 33: 73-81.

27. Zarembo JE, Douglas B, Valenta J, Weisbach JA (1974) Metabolites of mitragynine. Journal of Pharm Sci 63: 1407-1415.

28. Fattore L, Fratta W (2011) Beyond THC: the new generation of cannabinoid designer drugs, Front Behav Neurosci 5: 1-12

29. Matsumoto K, Takayama H, Narita M, Nakamura A, Suzuki M (2008) MGM-9 [(E)-Methyl 2-(3-ethyl-7a,12a- epoxyethanoxy)-9-fluoro1,2,3,4,6,7,12,12boctahydro- 8-methoxyindolo[2,3-a]quinolizin-2-yl)-3 methoxyacrylate], a derivative of the indole alkaloid mitragynine: a novel dualacting mu- and kappa-opioid agonist with potent antinociceptive and weak rewarding effects in mice. Neuropharmacology 55: 154-165.

30. M Tohda, S Thongpraditchote, K Matsumoto, Y Murakami, S Sakai (1997) Effects of mitragynine on cAMP formation mediated by delta-opiate receptors in NG108- 5 cells. Biol Pharma Bull 4: 338-340.

31. Kumarnsit E, Vongvatcharanon U, Keawpradub N, Intasaro P (2007) Foslike immunoreactivity in rat dorsal raphe nuclei induced by alkaloid extract of Mitragyna speciose. Neuroscience Letters 416: 128-132.

32. Hanapi NA, Azizi J, Ismail S, Mansor SM (2010) Evaluation of selected malaysian medicinal plants on phase I drug metabolizing enzymes, CYP2C9, CYP2D6 and CYP3A4 activities in vitro. Intern J Pharmacol 6: 494-499.

33. Le D, Goggin MM, Janis GC (2012) Analysis of mitragynine and metabolites in human urine for detecting the use of the psychoactive plant kratom. J Anal Toxicol 36: 616-625.

34. Meites J, Bruni F, Van Vugt DA, Smith AF (1979) Relation of endogenous opioid peptides and morphine to neuroendocrine functions. Life Sci 24: 13251336.

35. Kapp FG, Maurer HH, Auwärter V, Winkelmann M, Hermanns-Clausen M (2011) Intrahepatic cholestasis following abuse of powdered kratom (Mitragyna speciosa). J Med Toxicol 7: 227-231.

36. Roche et al. (2008) Kratom: A case of a legal high. Clinical Toxicology 46: 598.

37. Neerman MF, Frost RE, Deking J (2013) A drug fatality involving Kratom. J Forensic Sci 58: 278-279.

38. Idid S, Saad L, Yaacob H, Shahimi M (1998). Evaluation of analgesia induced by mitragynine, morphine and paracetamol in mice. ASEAN Review of Biodiversity and Environmental Conservation.

39. Eldridge W, Foster C, Wyble L (2018), Neonatal abstinence syndrome due to maternal kratom exposure, Pediatrics. 142: e20181839.
40. Matsumoto K, Yamamoto LT, Watanabe K, Yano S, Shan J, et al. (2005) Inhibitory effect of mitragynine, an analgesic alkaloid from Thai herbal medicine, on neurogenic contraction of the vas deferens. 78: 187-194.

41. DSM V, Diagnostic and Statistical Manual of Mental Disorders, Fifth Edition, American Psychiatric Association.

42. Havemann-Reinecke U (2011). Kratom and alcohol dependence: clinical symptoms, withdrawal treatment and pharmacological mechanisms - a case report. European Psychiatry 26: 1-50.

43. L.C. Thuan (1957). Addiction to Mitragyna speciose. Proceedings of the Alumni Association, Malaya, University Malaya, Kuala Lumpur 10: 322-324

44. Galbis-Reig D. A case report of Kratom Addiction and withdrawal. WMJWisoncin medical society. February 2016: 49-52.

45. Singh D, Müller CP, Vicknasingam BK (2014) Kratom (Mitragyna speciosa) dependence, withdrawal symptoms and craving in regular users. Drug Alcohol Depend 139: 132-137

46. Buresh M (2018). Treatment of Kratom Dependence with BuprenorphineNaloxone Maintenance. J Addict Med 12: 481-483.

47. Janchawee B, Keawpradub N, Chittrakarn S, Prasettho S, Wararatananurak et al (2007). A high-performance liquid chromatographic method for determination of mitragynine in serum and its application to a pharmacokinetic study in rats. Biomed Chromatogr 2: 176-183.

48. I. Wray (1907). Notes on the anti-opium remedy. Pharmaceutical Journal 78: 453

49. Sabetghadam A, Ramanathan S, Mansor SM. The evaluation of antinociceptive activity of alkaloid, methanolic, and aqueous extracts of Malaysian Mitragyna speciosa Korth leaves in rats. Pharmacognosy Res 2: 181-185.

50. Matsumoto K, Horie S, Takayama H, Ishikawa H, Aimi N, et al (2005). Antinociception, tolerance and withdrawal symptoms induced by 7-hydroxymitragynine, an alkaloid from the Thai medicinal herb Mitragyna speciose. Life Sci 78: 2-7.

51. Tsuchiya S, Miyashita S, Yamamoto M, Horie S, Sakai S, et al. Watanabe. Effect of mitragynine, derived from Thai folk medicine, on gastric acid secretion through opioid receptor in anesthetized rats. European Eur $\mathrm{J}$ Pharmacol 443: 185-188.

52. Gong F, H. Gu HP, Xu Q T, Kang WY (2012). Genus Mitragyna: ethnomedicinal uses and pharmacological studies. Phytopharmacology 3:263-272.

53. Chittrakarn S, Sawangjaroen K, Prasettho S, Janchawee B, Keawpradub N (2008). Inhibitory effects of kratom leaf extract (Mitragyna speciose Korth) on the rat gastro-intestinal tract. J Ethnopharmacol 116: 173-178.

54. Purintrapiban J, Keawpradub N, Kansenalak S, Chittrakarn S, Janchawee B, et al (2011). Study on glucose transport in muscle cells by extracts from Mitragyna speciosa (Korth) and mitragynine. Nat Prod Res 25: 1379- 1387.

55. Apryani E, Hidayat MT, Moklas MA, Fakurazi S, Idayu NF (2010). Effects of mitragynine from Mitragyna speciosa Korth leaves on working memory. J Ethnopharmacol 129: 357-360.

56. Hazim Al, Mustapha M, Mansor SM (2011). The effects on motor behaviour and short-term memory tasks in mice following an acute administration of Mitragyna speciosa alkaloid extract and mitragynine. Journal of Medicinal Plants Research 5-5810-5817.

57. Hagen EH, Sullivan RJ, Schmidt R, Morris G, Kempter R, et al (2009). Hammerstein Ecology and neurobiology of toxin avoidance and the paradox of drug reward. Neuroscience 160: 69-84.

58. Maurer $\mathrm{HH}$ (2010). Chemistry, pharmacology, and metabolism of emerging drugs of abuse. Ther Drug Monit 32: 544-549.

59. McWhirter L, Morris S. A case report if inpatient detoxification after kratom (Mitragyna speciosa) dependence. Eur Addict Res 16: 229-231.

60. Babu KM, McKurdy CR, Boyer EW (2008). Opioid receptors and legal highs: Salvia divinorum and kratom.Clinical Toxicology 46: 146-152. 